\title{
Understanding Contemplators' Knowledge and Awareness of the Physical Activity Guidelines
}

\author{
Katrina L. Piercy, Frances Bevington, Alison Vaux-Bjerke, Sandra Williams Hilfiker, \\ Sean Arayasirikul, and Elizabeth Y. Barnett
}

\begin{abstract}
Background: The Office of Disease Prevention and Health Promotion completed research to understand factors that could encourage Americans to follow the Physical Activity Guidelines for Americans, second edition, released in 2018. This study describes survey research assessing demographic characteristics that might be related to knowledge and awareness of the guidelines. Methods: An online survey of 2050 adult physical activity contemplators assessed knowledge of physical activity, awareness of the guidelines, and knowledge of dosage recommendations. Univariate and bivariate analyses were performed, and demographic differences in knowledge and awareness were analyzed using Pearson chi-square tests and Fisher exact tests. Results: Respondents had medium to high knowledge of physical activity, although knowledge varied significantly by socioeconomic factors. Knowledge of dosage recommendations was very low, with $2 \%$ and $3 \%$ of respondents correctly identifying recommended moderate- and vigorous-intensity doses, respectively. Only $22 \%$ were aware of the guidelines; awareness was greater among those with a higher education or income and those without a disability. Conclusions: These findings guided the development of the Office of Disease Prevention and Health Promotion's Move Your Way campaign and reinforced the need to raise awareness of the guidelines and promote behavior change among physical activity contemplatorsparticularly those from lower socioeconomic groups.
\end{abstract}

Keywords: exercise, national, promotion, campaign, recommendations

The Physical Activity Guidelines for Americans (the guidelines) outlines recommendations for the amount and types of physical activity necessary for good health based on the current scientific evidence. It includes specific physical activity dosages for youth and adults and additional recommendations for older adults and special populations. ${ }^{1}$ The first edition of the guidelines was released in 2008, ${ }^{2}$ and the second edition was released in November 2018. ${ }^{1,3}$ Although federal physical activity guidance is relatively recent, guidelines from professional organizations touting the health benefits of regular physical activity began emerging nearly 50 years ago. ${ }^{4}$

Despite the myriad benefits of physical activity, rates of physical inactivity remain high in the United States, with nearly $80 \%$ of adults being insufficiently active and a quarter of adults doing no leisure-time physical activity. ${ }^{5}$ In addition to lowering risk for all-cause mortality, coronary heart disease, type 2 diabetes, and hypertension, regular physical activity also reduces the risk of falls in older adults and positively affects brain health, bone health, weight status, cancer incidence, and physical function. ${ }^{1}$ In addition, adults generally overestimate their physical activity levels. ${ }^{6,7}$ Those who overestimate are less inclined to indicate they believe that physical activity has beneficial effects, and they express less intention to increase their physical activity compared with those who are realistic about their inactivity. ${ }^{7}$

One potential explanation for the low rates of physical activity is lack of awareness of the guidelines and lack of knowledge of

Piercy, Bevington, and Vaux-Bjerke are with the Office of Disease Prevention and Health Promotion, U.S. Department of Health and Human Services, Rockville, MD, USA. Hilfiker, Arayasirikul, and Barnett are with the CommunicateHealth, Inc., Rockville, MD, USA. Barnett (lizzie@communicatehealth.com) is corresponding author. dosage recommendations. In a 2009 survey, the percentage of adults who reported being aware of the guidelines was $36 \%$, and knowledge of the moderate-intensity aerobic physical activity dosage recommendation (at least $150 \mathrm{~min} / \mathrm{wk}$ ) was less than $1 \%{ }^{8}$ Knowledge of dosage recommendations for youth is also low; survey data collected in 2009 indicate that only about $10 \%$ of parents were knowledgeable about the amount of activity youth need (at least $60 \mathrm{~min}$ of moderate to vigorous physical activity each day). ${ }^{9}$

In their summary of Americans' awareness and knowledge of the first edition of the Physical Activity Guidelines for Americans, Kay et $\mathrm{al}^{8}$ concluded that the "nation needs more effective communication strategies to translate and disseminate physical activity guidelines." Therefore, when planning for the second edition of the guidelines, the Office of Disease Prevention and Health Promotion developed a corresponding communication campaign, Move Your Way. ${ }^{10}$ This campaign is grounded in the transtheoretical model (also known as stages of change) ${ }^{11}$ and aims to influence the behavior of physical activity contemplators.

This study focuses on the findings from a 2017 survey that assessed awareness of the guidelines, knowledge of the dosage recommendations, and knowledge of physical activity among American adult physical activity contemplators (ie, people who are not currently meeting the guidelines, but who are considering increasing their physical activity in the next $6 \mathrm{mo}$ ) as well as potential differences based on socioeconomic factors. This information was important to establish a baseline before launching the second edition of the guidelines to help assess any subsequent shift in awareness from Move Your Way campaign activities. The accompanying article, "The Move Your Way campaign: Encouraging contemplators and families to meet the recommendations from the Physical Activity Guidelines for Americans," 12 appearing in this issue, summarizes the findings from additional research that 
shaped the development of the Move Your Way campaign's products, messaging, and strategy.

\section{Methods}

The Office of Disease Prevention and Health Promotion worked with a health education and communication firm to conduct audience research with consumers. This research informed the development of a campaign and icon that would translate recommendations from the guidelines ${ }^{1}$ into accessible, actionable messages intended to move physical activity contemplators from the contemplation stage to preparation and action stages. This study focuses on one component of the research: an online survey of 2050 adults used to assess knowledge and awareness of the guidelines. Qualtrics (Provo, UT) fielded the survey for 3 weeks in May to June 2017 in preparation for the release of the second edition of the guidelines ${ }^{1}$ in late 2018 . The study was reviewed for compliance with the Paperwork Reduction Act and was approved by Chesapeake IRB (now Advarra) Review Board.

\section{Recruitment and Eligibility}

English-speaking adults aged 25-74 years were recruited by a Qualtrics online survey panel. Qualtrics, a market research firm, provides respondents for online surveys through an actively managed panel of members. Qualtrics recruits panel members through multiple channels, including social media, websites, and global partners. People in the pool of respondents have agreed to be contacted by market research services that seek survey respondents.

Research targeted 4 communities in states that are among those with the highest percentages of adults who report no leisure-time physical activity within the United States: Baltimore, MD; Chicago, IL; Jackson, MS; and Las Vegas, NV. ${ }^{13}$ Because of the lower number of respondents available in Jackson and Las Vegas, recruitment in those areas was expanded to include the entire states of Mississippi and Nevada, respectively. Only participants who were identified as physical activity contemplators (based on their answers to questions about their physical activity behavior and intention) and reported infrequent physical activity were eligible to be included in the study. Activity level was determined through screening questions that asked about frequency and duration of physical activity (eg, "How often do you get physical activity for at least 20 minutes a day?"). Participants were eligible to participate if they reported (1) infrequent physical activity and (2) the intention to increase physical activity in the next 6 months. Participant quotas were established for area (urban/ suburban/rural), sex, race/ethnicity, education, income, and disability to ensure that results reflected a demographically diverse sample. Eligible participants who completed the survey received Qualtrics reward points (worth less than \$5).

\section{Measures}

Demographic Characteristics. Demographic questions provided information on respondents' geographic area (rural, urban, or suburban); age; sex; race/ethnicity; education; income level; and parental status (not a parent to an adolescent, parent to 1 adolescent, parent to 2 adolescents, or parent to 3 or more adolescents); physical activity frequency $(0,1$, or 2-3 d/wk); and disability (either none or one or more physical or mental impairments).

Awareness of the Physical Activity Guidelines for Americans. A single yes/no question was used to assess awareness of the guidelines: Have you seen, heard, or read anything about federal physical activity guidelines?
Knowledge of Physical Activity. Four questions were used to gauge knowledge of physical activity: (1) What best describes what typically happens during moderate-intensity physical activity? (response options: your heart beats about the same as usual, and you can sing while doing the activity; your heart beats somewhat faster than usual, and you can talk while doing the activity; and your heart beats much faster than usual, and you cannot say more than a few words without pausing for a breath); (2) Choose the activity below that is typically considered moderate intensity (response options: jogging, brisk walking, fast swimming, and playing competitive basketball); (3) What best describes what typically happens during vigorous-intensity physical activity? (response options: your heart beats about the same as usual, and you can sing while doing the activity; your heart beats somewhat faster than usual, and you can talk while doing the activity; and your heart beats much faster than usual, and you cannot say more than a few words without pausing for a breath); and (4) Choose the activity below that is typically considered vigorous intensity (response options: running, walking the dog, gardening, and washing the dishes).

Knowledge of Dosage Recommendations. To assess knowledge of the dosage recommendations for adults, the following questions were asked about the moderate-intensity aerobic dose, the alternative vigorous-intensity aerobic dose, and musclestrengthening frequency: (1) What is the minimum amount of moderate-intensity physical activity the government recommends for adults to get big health benefits? (response options: 20 minutes a day, 3 or more days a week; 100 minutes spread out over a week; 30 minutes a day, 5 or more days a week; 60 minutes a day, 7 days a week; 150 minutes spread out over a week; or do not know/not sure); (2) What is the minimum amount of vigorous-intensity physical activity the government recommends for adults to get big health benefits? (response options: 15 minutes a day, 3 or more days a week; 75 minutes spread out over a week; 15 minutes a day, 5 or more days a week; 30 minutes a day, 3 or more days a week; 100 minutes spread out over a week; do not know/not sure); and (3) How many days a week of muscle-strengthening activity does the government recommend for adults to get big health benefits? (response options: at least 1 day a week, at least 2 days a week, at least 3 days a week, at least 4 days a week, and do not know/ not sure).

Overall Knowledge of Physical Activity and Dosage Recommendations. For questions related to knowledge of physical activity and knowledge of dosage recommendations, correct responses were assigned a single point and incorrect responses were assigned 0 points. Points for each respondent were totaled to create an individual composite knowledge score. Composite scores ranged from 0 to 7, with a mean of 3.51 and a median of 4 . Composite scores were divided into tertiles and categorized into low ( $0-3$ points, $\mathrm{n}=887$ ); medium ( 4 points, $\mathrm{n}=928$ ); and high $(5-7$ points, $n=235)$ scores of knowledge of physical activity and dosage recommendations.

\section{Data Analysis}

Data collected through the survey were analyzed with SPSS (SPSS Statistics for Windows, Released 2015, version 23.0; IBM Corp, Armonk, NY) using univariate and bivariate statistical analyses to describe sample demographics and aggregate responses to measured constructs. Differences in the data based on demographic or categorical outcomes were analyzed using Pearson chi-square tests and Fisher exact tests when appropriate. Awareness of the guidelines (aware and not aware) and the composite knowledge 
categories (low, medium, and high) were compared across parental status, education, income level, and disability status.

\section{Results}

Survey data from 2050 participants across 4 geographic locations were analyzed as described in the "Methods" section. Although attempts were made to gather approximately 500 participants from each location, response rates were lowest in Mississippi. Full descriptive characteristics are presented in Table 1. There was a range of ages represented but limited diversity in race/ ethnicity and gender. A majority of the respondents were female, were educated beyond a high school level, and did not have a child of adolescent age.

While individuals who indicate that they get at least 30 minutes of physical activity on at least 4 days a week were excluded from the study, $70 \%$ of the respondents reported doing physical activity 2 to 3 days per week.

\section{Awareness of the Physical Activity Guidelines for Americans}

Only $22 \%$ of participants indicated that they were aware of government physical activity guidelines. Overall, parents, individuals with a higher level of education, individuals with a higher income, and individuals without a disability had a greater awareness of the guidelines (Table 2). A greater proportion of individuals who reported being a parent of an adolescent were aware of the guidelines than those who were not a parent of an adolescent ( $27 \%$ vs $21 \% ; \chi^{2}=6.852, P=.01$ ). About $28 \%$ of participants with an education level of 4 years of college or more reported being aware of the guidelines compared with $17 \%$ of those with a high school education or less $\left(\chi^{2}=42.727, P<.001\right)$. Participants with greater household income were more likely to report being aware of the guidelines. For example, 33\% of respondents reporting a household income of $\$ 150,000$ or more were aware of the guidelines compared with $16 \%$ of those reporting a household income of less than $\$ 20,000\left(\chi^{2}=25.963, P<.001\right)$. Of the 254 participants who reported any disability, $84 \%$ were not aware of the guidelines. Compared with individuals without a disability, a significantly smaller proportion of participants who reported a disability were aware of the guidelines $\left(16 \%\right.$ vs $23 \% ; \chi^{2}=4.963$, $P=.03)$.

\section{Knowledge of Physical Activity}

On a positive note, respondents could correctly select a description of moderate- and vigorous-intensity physical activity (82\% for both) and were able to correctly choose the type of activity that is considered moderate intensity $(70 \%)$ or vigorous intensity $(95 \%)$.

\section{Knowledge of Dosage Recommendations}

Knowledge was very low for physical activity dosage recommendations (Table 3 ). For the moderate-intensity aerobic dosage, only $2 \%$ of respondents correctly chose " 150 minutes spread out over a week." However, a third of the respondents chose "30 minutes a day, 5 or more days a week," suggesting that there is some understanding of the recommendation in terms of total time. The same trend in responses was evident with the alternate dosage of vigorous-intensity aerobic activity; $3 \%$ correctly identified the time of 75 minutes, whereas $26 \%$ underestimated the amount by selecting 15 minutes a day, 3 days a week. Only $18 \%$ correctly identified the muscle-strengthening physical activity recommendation of 2 or more days a week, whereas $32 \%$ of respondents overestimated the recommended amount, choosing at least 3 days a week. An at a glance summary of results related to knowledge of the dosage recommendations is provided in Table 4 .

\section{Overall Knowledge of Physical Activity and Dosage Recommendations}

Overall, respondents had medium to high knowledge of physical activity and dosage recommendations (Table 5), as indicated with a composite score of 4 or higher. Knowledge levels varied significantly by socioeconomic factors (Table 5). Nearly half or more of each racial/ethnic minority group had a low level of knowledge compared with $39 \%$ of white respondents $\left(\chi^{2}=64.483\right.$, $P<.001)$. Over half of respondents with an education level of high school or less had a low level of knowledge compared with $40 \%$ of respondents with 4 years of college or more. Only $9 \%$ of respondents with an education level of high school or less had a high level of knowledge compared with $13 \%$ of respondents with 4 years of college or more $\left(\chi^{2}=23.423, P=.001\right)$. Participants with a greater household income reported higher levels of knowledge. For example, $15 \%$ of respondents reporting a household income of $\$ 150,000$ or more had a high level of knowledge compared with $7 \%$ of those reporting a household income of less than $\$ 20,000$. Similarly, $54 \%$ of respondents reporting a household income of less than $\$ 20,000$ had a low level of knowledge compared with $34 \%$ of those with a household income of $\$ 150,000$ or more $\left(\chi^{2}=\right.$ 42.606, $P<.001)$. Participants with no reported disability had higher levels of knowledge than those who reported having a disability. For example, $12 \%$ of respondents who did not report a disability had a high level of knowledge compared with $7 \%$ of those who reported a disability. Conversely, a greater proportion of those who reported a disability had a low level of knowledge than their counterparts with no reported disability (49\% vs $42 \%$ ) $\left(\chi^{2}=7.370, P=.03\right)$.

\section{Discussion}

The survey gathered information on the following research questions about contemplators: (1) What is the level of physical activity knowledge? (2) What is the level of awareness of the Physical Activity Guidelines for Americans? and (3) What is the level of knowledge about physical activity dosage recommendations from the guidelines? In addition, this survey explored how socioeconomic factors are related to differences in overall knowledge of physical activity and dosage recommendations. Overall, the findings, while somewhat disappointing from a public health perspective, provided a valuable foundation to guide development of the Move Your Way campaign. They further reinforced the need for a comprehensive communication campaign to help raise awareness of the guidelines and promote behavior change, especially for socioeconomic groups that had even lower knowledge and awareness compared with other groups.

\section{Awareness of the Physical Activity Guidelines for Americans}

Awareness of federal recommendations for physical activity appears to be decreasing. In a previous study conducted in $2009,36 \%$ of respondents reported being aware of federal recommendations 
Table 1 Demographic Characteristics Summary $(\mathrm{N}=\mathbf{2 0 5 0})^{\mathrm{a}}$

\begin{tabular}{|c|c|c|}
\hline & $\mathbf{n}$ & $\%$ \\
\hline \multicolumn{3}{|l|}{ Location } \\
\hline Baltimore & 560 & 27 \\
\hline Chicago & 600 & 29 \\
\hline Mississippi & 345 & 17 \\
\hline Nevada & 545 & 27 \\
\hline \multicolumn{3}{|l|}{ Area } \\
\hline Urban & 634 & 31 \\
\hline Suburban & 1142 & 56 \\
\hline Rural & 274 & 13 \\
\hline \multicolumn{3}{|l|}{ Age, y } \\
\hline $25-34$ & 539 & 26 \\
\hline $35-44$ & 451 & 22 \\
\hline $45-54$ & 351 & 17 \\
\hline $55-64$ & 389 & 19 \\
\hline $65-74$ & 320 & 16 \\
\hline \multicolumn{3}{|l|}{ Sex } \\
\hline Male & 600 & 29 \\
\hline Female & 1450 & 71 \\
\hline \multicolumn{3}{|l|}{ Race/ethnicity } \\
\hline White & 1518 & 74 \\
\hline Hispanic/Latino & 126 & 6 \\
\hline Black/African American & 262 & 13 \\
\hline American Indian/Alaska Native & 11 & 1 \\
\hline Asian/Pacific Islander & 66 & 3 \\
\hline Multiracial or other & 67 & 3 \\
\hline \multicolumn{3}{|l|}{ Education } \\
\hline High school or less & 300 & 15 \\
\hline Some college & 478 & 23 \\
\hline Associate degree & 266 & 13 \\
\hline 4-y college or more & 1006 & 49 \\
\hline \multicolumn{3}{|l|}{ Income } \\
\hline$<\$ 20,000$ & 217 & 11 \\
\hline$\$ 20,000-\$ 34,999$ & 258 & 13 \\
\hline$\$ 35,000-\$ 49,999$ & 303 & 15 \\
\hline$\$ 50,000-\$ 74,999$ & 467 & 23 \\
\hline$\$ 75,000-\$ 99,999$ & 332 & 16 \\
\hline$\$ 100,000-\$ 149,999$ & 304 & 15 \\
\hline$\$ 150,000-\$ 199,999$ & 94 & 5 \\
\hline$\$ 200,000$ or more & 75 & 4 \\
\hline \multicolumn{3}{|l|}{ Parent status } \\
\hline Not a parent to an adolescent & 1672 & 82 \\
\hline 1 adolescent & 247 & 12 \\
\hline 2 adolescents & 96 & 5 \\
\hline 3 or more adolescents & 35 & 2 \\
\hline \multicolumn{3}{|l|}{ Physical activity frequency, $\mathrm{d} / \mathrm{wk}$} \\
\hline 0 & 99 & 5 \\
\hline 1 & 515 & 25 \\
\hline $2-3$ & 1436 & 70 \\
\hline
\end{tabular}

(continued)
Table 1 (continued)

\begin{tabular}{lcc}
\hline & $\mathbf{n}$ & $\%$ \\
\hline $\begin{array}{l}\text { Disability } \\
\quad \begin{array}{l}\text { One or more disabilities or } \\
\text { impairments (hearing, vision, } \\
\text { decision making, and physical) }\end{array}\end{array}$ & 12 \\
$\quad$ No disability & 254 & \\
\hline a Percentages have been rounded and may not sum to 100. & 1796 & 88 \\
\hline
\end{tabular}

Table 2 Differences in Awareness of Physical Activity Guidelines for Americans ( $N=2050)$

\begin{tabular}{|c|c|c|c|c|c|c|}
\hline \multirow[b]{2}{*}{ Variable } & \multicolumn{2}{|c|}{$\begin{array}{c}\text { Aware } \\
(n=450)\end{array}$} & \multicolumn{2}{|c|}{$\begin{array}{l}\text { Not aware } \\
(n=1600)\end{array}$} & \multirow[b]{2}{*}{$\chi^{2}$} & \multirow{2}{*}{$\begin{array}{c}P \\
\text { value }\end{array}$} \\
\hline & $\mathbf{n}$ & $\%$ & $\mathrm{n}$ & $\%$ & & \\
\hline \multicolumn{7}{|l|}{ Parent status } \\
\hline Yes & 102 & 27.0 & 276 & 73.0 & 6.852 & .01 \\
\hline No & 348 & 20.8 & 1324 & 79.2 & & \\
\hline \multicolumn{7}{|l|}{ Education } \\
\hline High school or less & 50 & 16.7 & 250 & 83.3 & 42.727 & $<.001$ \\
\hline Some college & 70 & 14.6 & 408 & 85.4 & & \\
\hline Associate degree & 49 & 18.4 & 217 & 81.6 & & \\
\hline 4-y college or more & 281 & 27.9 & 725 & 72.1 & & \\
\hline \multicolumn{7}{|l|}{ Income } \\
\hline$<\$ 20,000$ & 35 & 16.1 & 182 & 83.9 & 25.963 & $<.001$ \\
\hline$\$ 20,000-\$ 34,999$ & 48 & 18.6 & 210 & 81.4 & & \\
\hline$\$ 35,000-\$ 49,999$ & 60 & 19.8 & 243 & 80.2 & & \\
\hline$\$ 50,000-\$ 74,999$ & 88 & 18.8 & 379 & 81.2 & & \\
\hline$\$ 75,000-\$ 99,999$ & 86 & 25.9 & 246 & 74.1 & & \\
\hline$\$ 100,000-\$ 149,999$ & 78 & 25.7 & 226 & 74.3 & & \\
\hline$\$ 150,000$ or more & 55 & 32.5 & 114 & 67.5 & & \\
\hline \multicolumn{7}{|l|}{ Disability } \\
\hline $\begin{array}{l}\text { Any reported } \\
\text { disability }\end{array}$ & 42 & 16.5 & 212 & 83.5 & 4.963 & .03 \\
\hline No reported disability & 408 & 22.7 & 1388 & 77.3 & & \\
\hline
\end{tabular}

for physical activity. ${ }^{8}$ In contrast, only $22 \%$ of participants in this survey were aware of federal physical activity recommendations. Because the first edition of the Physical Activity Guidelines for Americans was released in 2008, the higher awareness captured in the 2009 study may be due in part to peak communication and dissemination efforts during that time. A promotional campaign, Be Active Your Way, was developed and disseminated with the first edition of the guidelines ${ }^{2}$; however, it was a small-scale campaign and mainly focused on the initial year after the guidelines was released. ${ }^{14}$ In addition, our study excluded individuals already meeting the guidelines, suggesting that awareness may be lower in those who most need to know about the guidelines to help inform lifestyle choices.

\section{Knowledge of Dosage Recommendations}

Knowledge of the specific aerobic physical activity dosage necessary for health benefits remains low. This study noted that only $2 \%$ of participants correctly selected "150 minutes spread out over 
Table 3 Knowledge of Physical Activity and Dosage Recommendations, Item by Item $(\mathrm{N}=\mathbf{2 0 5 0})$

\begin{tabular}{lcc}
\hline Variables & $\mathbf{n}$ & $\%$ \\
\hline Item 1-Moderate-intensity physical activity definition & \\
Correct & 1673 & 81.6 \\
Incorrect & 377 & 18.4 \\
Item 2-Identify moderate-intensity physical activity & \\
Correct & 1429 & 69.7 \\
Incorrect & 621 & 30.3 \\
Item 3-Moderate-intensity physical activity guideline & \\
Correct & 34 & 1.7 \\
Incorrect & 2016 & 98.3 \\
Item 4-Vigorous-intensity physical activity definition & \\
Correct & 1676 & 81.8 \\
Incorrect & 374 & 18.2 \\
Item 5-Identify vigorous-intensity physical activity & \\
Correct & 1953 & 95.3 \\
Incorrect & 97 & 4.7 \\
Item 6-Vigorous-intensity physical activity guideline & \\
Correct & 60 & 2.9 \\
Incorrect & 1990 & 97.1 \\
Item 7-Muscle-strengthening physical activity guideline & \\
Correct & 363 & 17.7 \\
Incorrect & 1687 & 82.3 \\
\hline
\end{tabular}

a week," which is similar to findings from Kay et $\mathrm{al}^{8}$ of $0.56 \%$. Interestingly, more contemplators seem to understand the moderate-intensity aerobic physical activity dosage recommendation from the guidelines in terms of days per week (ie, 30 minutes a day, 5 or more days a week) than in terms of total time (ie, 150 minutes a week). In this study, 34\% selected "30 minutes a day, 5 or more days a week," which is consistent with the findings from Kay et al, ${ }^{8}$ who documented that $33.3 \%$ chose the same response. Perhaps this is because of earlier physical activity recommendations that emphasized a daily time allotment, with
30 minutes being more memorable than 150 minutes. The 1995 American College of Sports Medicine/Centers for Disease Control and Prevention physical activity recommendations suggested that "every US adult should accumulate 30 minutes or more of moderate-intensity physical activity on most, preferably all, days of the week." 3 And several other studies have documented an awareness of 30 minutes per day, 5 days per week. ${ }^{15-17}$ In addition, both in this survey and Kay et al's ${ }^{8}$ survey, approximately $25 \%$ of respondents indicated that they did not know or were not aware of the recommended amount of physical activity. Thirty minutes of activity, 5 days a week is equivalent to 150 minutes a week, so technically, the response selected by a third of participants is one way to meet the guidelines but is not the specific adult dosage guideline. The 2018 Physical Activity Guidelines Advisory Committee clearly confirmed the 2008 Physical Activity Guidelines for Americans ${ }^{2}$ in its Scientific Report, ${ }^{18}$ which recommended a weekly target rather than a daily amount. This recommendation was incorporated into the second edition of the guidelines, ${ }^{1}$ which recommends a target weekly range rather than a daily amount. The recommendation for a weekly accumulation of physical activity is based on the current scientific evidence, ${ }^{18}$ which suggests benefits can be accrued in a variety of ways. Ideally, physical activity is spread throughout the week, ${ }^{1}$ but benefits can also come to those who concentrate physical activity across just a few days, often described as "weekend warriors.",

\section{Overall Knowledge of Physical Activity and Dosage Recommendations}

Prior research has shown that individuals meeting the physical activity recommendations are more likely to be knowledgeable about them. ${ }^{15,20}$ This study focused only on contemplators-as those who reported getting regular physical activity were excluded, the focus was on those that would potentially benefit most from targeted messages that support and encourage them to meet the guidelines. Only $12 \%$ of participants had a high score for knowledge of physical activity and dosage recommendations, quantified as correctly answering 5 to 7 questions. The remaining participants were split between low scores (correctly answering

Table 4 Knowledge of the Dosage Recommendations at a Glance $(\mathrm{N}=\mathbf{2 0 5 0})$

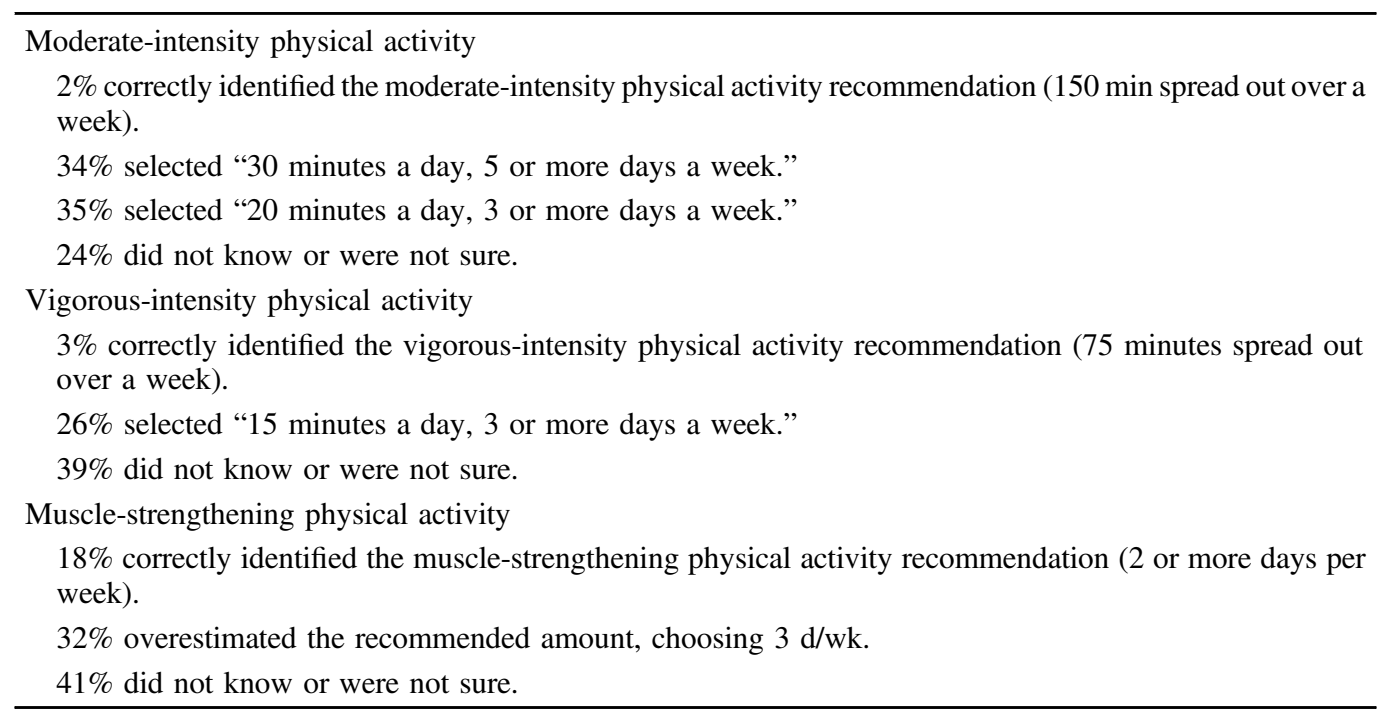


Table 5 Levels of Knowledge of Physical Activity and Dosage Recommendations by Demographic Variables of Interest, Row Percentages $(\mathrm{N}=\mathbf{2 0 5 0})$

\begin{tabular}{|c|c|c|c|c|c|c|c|c|}
\hline \multirow{2}{*}{$\begin{array}{l}\text { Variable } \\
\text { Composite categorical (3 levels) }\end{array}$} & \multicolumn{5}{|c|}{$\mathrm{n}$} & \multicolumn{3}{|c|}{$\%$} \\
\hline & & & & & & & & \\
\hline Low (scores $0-3$ ) & \multicolumn{5}{|c|}{887} & \multicolumn{3}{|c|}{43.3} \\
\hline Medium (score 4) & \multicolumn{5}{|c|}{928} & \multicolumn{3}{|c|}{45.3} \\
\hline High (scores 5-7) & \multicolumn{4}{|c|}{235} & & \multicolumn{3}{|c|}{11.5} \\
\hline & \multicolumn{2}{|c|}{ Low } & \multicolumn{2}{|c|}{ Medium } & \multicolumn{2}{|c|}{ High } & \multirow[t]{2}{*}{$\chi^{2}$} & \multirow[t]{2}{*}{$P$ value } \\
\hline Variables & $\mathrm{n}$ & $\%$ & $\mathrm{n}$ & $\%$ & $\mathrm{n}$ & $\%$ & & \\
\hline \multicolumn{9}{|l|}{ Race/ethnicity } \\
\hline White & 587 & 38.7 & 740 & 48.7 & 191 & 12.6 & \multirow[t]{6}{*}{64.483} & \multirow[t]{6}{*}{$<.001$} \\
\hline Hispanic/Latino & 83 & 65.9 & 36 & 28.6 & 7 & 5.6 & & \\
\hline Black/African American & 136 & 51.9 & 97 & 37.0 & 29 & 11.1 & & \\
\hline American Indian/Alaska Native & 7 & 63.6 & 3 & 27.3 & 1 & 9.1 & & \\
\hline Asian/Pacific Islander & 42 & 63.6 & 23 & 34.8 & 1 & 1.5 & & \\
\hline Multiracial or other & 32 & 47.8 & 29 & 43.3 & 6 & 9.0 & & \\
\hline \multicolumn{9}{|l|}{ Education } \\
\hline High school or less & 155 & 51.7 & 118 & 39.3 & 27 & 9.0 & \multirow[t]{4}{*}{23.423} & \multirow[t]{4}{*}{.001} \\
\hline Some college & 204 & 42.7 & 232 & 48.5 & 42 & 8.8 & & \\
\hline Associate degree & 129 & 48.5 & 105 & 39.5 & 32 & 12.0 & & \\
\hline 4-y college or more & 399 & 39.7 & 473 & 47.0 & 134 & 13.3 & & \\
\hline \multicolumn{9}{|l|}{ Income } \\
\hline$<\$ 20,000$ & 118 & 54.4 & 85 & 39.2 & 14 & 6.5 & \multirow[t]{7}{*}{42.606} & \multirow[t]{7}{*}{$<.001$} \\
\hline$\$ 20,000-\$ 34,999$ & 138 & 53.5 & 95 & 36.8 & 25 & 9.7 & & \\
\hline$\$ 35,000-\$ 49,999$ & 140 & 46.2 & 129 & 42.6 & 34 & 11.2 & & \\
\hline$\$ 50,000-\$ 74,999$ & 196 & 42.0 & 212 & 45.4 & 59 & 12.6 & & \\
\hline$\$ 75,000-\$ 99,999$ & 128 & 38.6 & 168 & 50.6 & 36 & 10.8 & & \\
\hline$\$ 100,000-\$ 149,999$ & 110 & 36.2 & 152 & 50.0 & 42 & 13.8 & & \\
\hline$\$ 150,000$ or more & 57 & 33.7 & 87 & 51.5 & 25 & 14.8 & & \\
\hline \multicolumn{9}{|l|}{ Disability } \\
\hline Any reported disability & 125 & 49.2 & 111 & 43.7 & 18 & 7.1 & \multirow[t]{2}{*}{7.370} & \multirow[t]{2}{*}{.03} \\
\hline No reported disability & 762 & 42.4 & 817 & 45.5 & 217 & 12.1 & & \\
\hline
\end{tabular}

0-3 questions) and medium scores (correctly answering 4 questions).

\section{Socioeconomic Factors, Overall Knowledge of Physical Activity and Dosage, and Awareness of the Physical Activity Guidelines for Americans}

Currently, less than $25 \%(24.3 \%)$ of adults meet the aerobic and muscle-strengthening physical activity recommendations. ${ }^{21}$ However, there are disparities across age, gender, race, education level, disability, and income. ${ }^{21}$ Many factors influence how active an individual is and how motivated he or she is to become more active. One contributing factor is knowledge or awareness of the benefits of regular physical activity. ${ }^{15,20}$ Individuals of racial/ ethnic minorities, individuals with less than a high school education, those with a lower household income, and those with a disability had lower overall knowledge of physical activity and dosage recommendations than white respondents, individuals with 4 years of college or more, those with a household income over $\$ 150,000$, and those without a disability, respectively. The same pattern was true for awareness of the guidelines-individuals with less than a high school education, those with a lower household income, and those with a disability were less likely to be aware of the guidelines than individuals with 4 years of college or more, those with a household income over $\$ 150,000$, and those without a disability, respectively.

Previous findings suggest that socioeconomic status ${ }^{22-24}$ and racial/ethnic factors ${ }^{22,25}$ play a role in physical activity levels. Demographics also impact knowledge of physical activity. ${ }^{17,26}$ Health disparities for racial/ethnic minority groups may be explained by physical activity level alone. ${ }^{22}$ Higher level of education, ${ }^{24}$ better health status, ${ }^{27}$ and higher socioeconomic status have all been associated with higher physical activity levels. In addition, people from racial/ethnic minority groups as well as those with limited formal education are often less aware of the health benefits of physical activity than are people from nonminority groups and people with more education. ${ }^{23}$

Health promotion campaigns, especially those based in theory, have been shown to be effective interventions for behavior change. ${ }^{18,28,29}$ Therefore, physical activity communication campaigns that consider socioeconomic factors could help address disparities in levels of knowledge about physical activity to 
improve physical activity behavior. Most physical activityfocused campaigns have not targeted specific demographic groups ${ }^{29}$ More research is necessary to determine whether addressing knowledge disparities could reduce disparities in levels of physical activity.

\section{Limitations}

Survey data were collected from physical activity contemplators aged 25-74 years in 4 diverse geographic locations across the United States. Results are neither generalizable to the larger population, nor do they include a nationally representative sample. In contrast, the HealthStyles survey used by Kay et al ${ }^{8}$ in 2009 was sent to adults aged 18 years and older and was poststratified and weighted to create a nationally representative sample. ${ }^{30}$ Participants for this survey were recruited through a Qualtrics online panel, suggesting that they are more likely to be computer and web literate. People with lower incomes and lower education levels tend to be underrepresented among Internet users. ${ }^{31}$

\section{Conclusions}

This study was unique in that it focused only on physical activity contemplators and examined how socioeconomic factors may be related to overall knowledge of physical activity and dosage and awareness of the guidelines. The Office of Disease Prevention and Health Promotion completed the research summarized here to inform the Move Your Way campaign, which was developed to promote the recommendations from the second edition of the Physical Activity Guidelines for Americans. As discussed, the 30 minutes a day recommendation appears to be more memorable than a weekly target. This point was considered extensively during development of the Move Your Way campaign, as was how best to deliver the key guidelines for specific populations. Given feedback from this survey and focus groups conducted to inform the Move Your Way campaign, messages that deemphasize the target of 150 minutes and instead focus on simple ways to incorporate more physical activity into daily life may be more effective.

This survey confirmed a strong need for more effective communication strategies to translate and disseminate physical activity guidance, an important finding for those whose work relates to physical activity-related campaigns, interventions, communications, or education. These findings also suggest that physical activity communication campaigns that consider socioeconomic factors could help address disparities in levels of knowledge about physical activity to improve physical activity behavior. Researchers may be interested in further studying the relationship between knowledge disparities and disparities in a health promoting behavior, such as physical activity.

\section{Acknowledgments}

The authors thank the survey participants, whose willingness to take the survey made this study possible. The authors are grateful to Kate Olscamp (Office of Disease Prevention and Health Promotion) and Corinne Berry, Dena Fisher, Morgan Griffin, and Janel Schuh (CommunicateHealth, Inc.), who provided technical assistance, comments, and suggestions throughout the course of the study. The authors also would like to thank Blythe Miller, Laura Willwerth, and Andrea Mongler (CommunicateHealth, Inc.) for their writing assistance. This work was supported by the U.S. Department of Health and Human Services.

\section{References}

1. U.S. Department of Health and Human Services. Physical Activity Guidelines for Americans. 2nd ed. https://health.gov/paguidelines/ second-edition/. Published 2018. Accessed May 20, 2019.

2. U.S. Department of Health and Human Services. 2008 Physical Activity Guidelines for Americans. https://health.gov/paguidelines/ 2008/. Published 2008. Accessed May 20, 2019.

3. Pate RR, Pratt M, Blair SN, et al. Physical activity and public health. A recommendation from the Centers for Disease Control and Prevention and the American College of Sports Medicine. JAMA. 1995;273:402-407. https://jamanetwork.com/journals/jama/articleabstract/386766. Accessed May 20, 2019. PubMed ID: 7823386 doi:10.1001/jama.1995.03520290054029

4. American College of Sports Medicine. American College of Sports Medicine position statement on the recommended quantity and quality of exercise for developing and maintaining fitness in healthy adults. Med Sci Sports. 1978;10(3):vii-x.

5. U.S. Department of Health and Human Services, Office of Disease Prevention and Health Promotion. Healthy People 2020 Topics and Objectives: Physical Activity. https://www.healthypeople.gov/2020/ topics-objectives/topic/physical-activity/objectives. Updated May 20, 2019. Accessed May 20, 2019.

6. Troiano R, Berrigan D, Dodd K, Mâsse L, Tilert T, McDowell M. Physical activity in the United States measured by accelerometer. Med Sci Sports Exerc. 2008;40(1):181-188. PubMed ID: 18091006 doi:10.1249/mss.0b013e31815a51b3

7. Godino JG, Watkinson C, Corder K, Sutton S, Griffin SJ, van Sluijs EM. Awareness of physical activity in healthy middle-aged adults: a cross-sectional study of associations with sociodemographic, biological, behavioural, and psychological factors. BMC Public Health. 2014;14:421. PubMed ID: 24886612 doi:10.1186/14712458-14-421

8. Kay MC, Carroll DD, Carlson SA, Fulton JE. Awareness and knowledge of the 2008 Physical Activity Guidelines for Americans. J Phys Act Health. 2014;11(4):693-698. PubMed ID: 23493071 doi:10.1123/jpah.2012-0171

9. DeBastiani SD, Carroll DD, Cunningham M, Lee S, Fulton J. Awareness and knowledge of the youth 2008 Physical Activity Guidelines for Americans. J Phys Act Health. 2014;11(3):495501. PubMed ID: 23493091 doi:10.1123/jpah.2012-0207

10. U.S. Department of Health and Human Services, Office of Disease Prevention and Health Promotion. Move Your Way. https://health. gov/moveyourway/. Accessed May 20, 2019.

11. Prochaska JO. Transtheoretical model of behavior change. In: Gellman MD, Turner JR, eds. Encyclopedia of Behavioral Medicine. New York, NY: Springer; 2013:1997-2000.

12. Bevington F, Piercy KL, Olscamp K, Hilfiker SW, Fisher DG, Barnett EY. The Move Your Way campaign: encouraging contemplators and families to meet the recommendations from the Physical Activity Guidelines for Americans J Phys Act Health. 2020;17(4):397-403. doi:10.1123/jpah.2019-0395

13. Centers for Disease Control and Prevention. 2014 State Indicator Report on Physical Activity. https://www.cdc.gov/physicalactivity/ downloads/pa_state_indicator_report_2014.pdf. Published July 2014. Accessed May 20, 2019.

14. Piercy KL, Loughrey K, Wargo JD. Communication strategies to promote the 2008 Physical Activity Guidelines for Americans. In: Pate RR, Buchner DM, eds. Implementing Physical Activity Strategies: Put the National Physical Activity Plan into Action with 42 Proven Programs. Champaign, IL: Human Kinetics; 2014: 91-101. 
15. Bennett GG, Wolin KY, Puleo EM, Mâsse LC, Atienza AA. Awareness of national physical activity recommendations for health promotion among US adults. Med Sci Sports Exerc. 2009; 41(10):1849-1855. PubMed ID: 19727030 doi:10.1249/MSS. 0b013e3181a52100

16. Morrow JR, Krzewinski-Malone JA, Jackson AW, Bungum TJ, Fitzgerald SJ. American adults' knowledge of exercise recommendations. Res Q Exerc Sport. 2004;75:231-237. PubMed ID: 15487287 doi:10.1080/02701367.2004.10609156

17. Moore LV, Fulton J, Kruger J, McDivitt J. Knowledge of physical activity guidelines among adults in the United States, HealthStyles 2003-2005. J Phys Act Health. 2010;7(2):141-149. PubMed ID: 20484752 doi:10.1123/jpah.7.2.141

18. 2018 Physical Activity Guidelines Advisory Committee. 2018 Physical Activity Guidelines Advisory Committee Scientific Report. https://health.gov/paguidelines/second-edition/report. Published February 2018. Accessed June 5, 2019.

19. O'Donovan G, Lee IM, Hamer M, Stamatakis E. Association of "weekend warrior" and other leisure time physical activity patterns with risks for all-cause, cardiovascular disease, and cancer mortality. JAMA Intern Med. 2017;177(3):335-342. doi:10.1001/ jamainternmed.2016.8014

20. Abula K, Gröpel P, Chen K, Beckmann J. Does knowledge of physical activity recommendations increase physical activity among Chinese college students? Empirical investigations based on the transtheoretical model. J Sport Health Sci. 2018;7(1):77-82. PubMed ID: 30356484 doi:10.1016/j.jshs.2016.10.010

21. U.S. Department of Health and Human Services, Office of Disease Prevention and Health Promotion. Healthy People 2020 topics and objectives: physical activity, PA-2.4. https://www.healthypeople. gov/node/5072/data_details. Updated May 20, 2019. Accessed May 20, 2019.

22. Meyer OL, Castro-Schilo L, Agular-Gaxiola S. Determinants of mental health and self-rated health: a model of socioeconomic status, neighborhood safety, and physical activity. Am J Public Health. 2014; 104(9):1734-1741. PubMed ID: 25033151 doi:10.2105/AJPH.2014. 302003
23. Waters EA, Hawkins E. Awareness of health outcomes associated with insufficient physical activity and associations with physical activity intentions and behavior. J Health Commun. 2018;23(7), 634-642. PubMed ID: 30089442 doi:10.1080/10810730.2018.1500658

24. Harper S, Lynch J. Trends in socioeconomic inequalities in adult health behaviors among U.S. states, 1990-2004. Public Health Rep. 2007;122: 177-189. PubMed ID: 17357360 doi:10.1177/003335490712200207

25. Neighbors CJ, Marquez DX, Marcus BH. Leisure-time physical activity disparities among Hispanic subgroups in the United States. Am J Public Health. 2008;98(8):1460-1464. PubMed ID: 18048795 doi:10.2105/AJPH.2006.096982

26. Knox ECL, Esliger DW, Biddle SJH, Sherar LB. Lack of knowledge of physical activity guidelines: can physical activity promotion campaigns do better? BMJ Open. 2013;3(12):e003633. PubMed ID: 24319271 doi:10.1136/bmjopen-2013-003633

27. Garber CE, Allsworth JE, Marcus BH, Hesser J, Lapane KL. Correlates of the stages of change for physical activity in a population survey. Am J Public Health. 2008;98(5):897-904. PubMed ID: 18381988 doi:10.2105/AJPH.2007.123075

28. The Community Guide. Health communication and social marketing: campaigns that include mass media and health-related product distribution. https://www.thecommunityguide.org/findings/healthcommunication-and-social-marketing-campaigns-include-mass-mediaand-health-related. Updated August 22, 2018. Accessed January 17, 2019.

29. Bergeron CD, Tanner AH, Friedman DB, et al. Physical activity communications: a scoping review of the literature. Health Promot Pract. 2019;20(3):344-353. PubMed ID: 30832516 doi:10.1177/ 1524839919834272

30. Pollard WE. Joint Statistical Meetings-Section on Health Policy Statistics. Use of Consumer Panel Survey Data for Public Health Communication Planning: an Evaluation of Survey Results. http:// citeseerx.ist.psu.edu/viewdoc/download?doi=10.1.1.380.1725\&rep= rep1\&type $=$ pdf. Published 2002. Accessed May 20, 2019.

31. Pew Research Center. Collecting survey data. https://www. pewresearch.org/methods/u-s-survey-research/collecting-survey-data/ \#internet-surveys. Accessed May 20, 2019. 